\title{
Triatoma vandae sp. n. do Complexo oliveirai Encontrada no Estado de Mato Grosso, Brasil (Hemiptera: Reduviidae: Triatominae)
}

\author{
Rodolfo U Carcavallo/ ${ }^{+}$, José Jurberg, Dayse da Silva Rocha, Cleber Galvão, \\ François Noireau*, Herman Lent**
}

\begin{abstract}
Laboratório Nacional e Internacional de Referência em Taxonomia de Triatomíneos, Departamento de Entomologia, Instituto Oswaldo Cruz-Fiocruz, Av. Brasil 4365, 21045-900 Rio de Janeiro, RJ, Brasil *Institut de Recherche pour le Développement, UR016, Paris, France **Centro de Ciências Biológicas, Universidade Santa Úrsula, Rio de Janeiro, RJ, Brasil
\end{abstract}

Triatoma vandae sp.n. of the oliveirai complex from the State of Mato Grosso, Brazil (Hemiptera: Reduviidae: Triatominae) - There are several specific complexes belonging to the genus Triatoma Laporte, 1832, which are generally associated to specific geographic areas. Recent publications have linked the oliveirai complex to ecosystems of Mato Grosso, which are also present in other Brazilian states and even in other bordering countries as eastern Paraguay. The study of the abundant material collected during the last years allowed the description of several new species of the oliveirai complex: T. jurbergi Carcavallo, Galvão \& Lent, 1998; T. baratai Carcavallo \& Jurberg, 2000 and T. klugi Carcavallo, Jurberg, Lent \& Galvão, 2001. Another new species belonging to the same complex is described here as T. vandae sp.n. It originates from the state of Mato Grosso, and has been reared in the insectary of the Laboratório Nacional e Internacional de Referência em Taxonomia de Triatomíneos, Departamento de Entomologia, Instituto Oswaldo Cruz-Fiocruz, Rio de Janeiro.

Key words: Triatoma vandae sp. n. - oliveirai complex - Triatominae - Chagas disease - Mato Grosso - Brazil

As numerosas espécies do gênero Triatoma Laporte, 1832 podem ser reagrupadas já que muitas possuem caracteres morfológicos, etológicos e/ou genéticos semelhantes. Estes grupos de espécies semelhantes são nomeados "complexos específicos" (Usinger et al. 1966, Lent \& Wygodzinsky 1979) e foram estudados recentemente em sua filogenia e possível origem geográfica (Carcavallo et al. 1999, 2000).

Nos últimos anos, o Laboratório Nacional e Internacional de Referência em Taxonomia de Triatomíneos recebeu farto material dos ecossistemas da Região CentroOeste do Brasil, especialmente dos Estados de Mato Grosso, Mato Grosso do Sul e outros vizinhos com caracteres ecológicos semelhantes. Grande parte desse material pertencia ao "complexo oliveirai" que é composto pela espécie nominotípica Triatoma oliveirai Neiva, Pinto \& Lent, 1939 além de T. matogrossensis Leite \& Barbosa, 1953; T. williami Galvão, Souza \& Lima, 1965 e T. guazu Lent \& Wygodzinsky, 1979. O abundante material coletado e criado no laboratório permitiu a descrição de três novas espécies (T. jurbergi Carcavallo, Galvão \& Lent, 1998; T. baratai Carcavallo \& Jurberg, 2000 e T. klugi Carcavallo, Jurberg, Lent \& Galvão, 2001) que foram integradas ao complexo. Recentemente Galvão et al. (2001) incluíram $T$.

Com auxílio do CNPq, da Faperj e do Convênio Funasa/Fiocruz

+Autor de contato. Fax: +55-21-2573.4468

E-mail: carcaval@gene.dbbm.fiocruz.br

Recebido em 28 de agosto de 2001

Aceito em 21 de março de 2002 deaneorum Galvão, Souza \& Lima, 1967 no complexo oliveirai, que está atualmente constituído de oito espécies. No presente trabalho é descrita uma nova espécie do complexo oliveirai, proveniente do município de Itiquira, Mato Grosso, Brasil.

\section{MATERIAIS E MÉTODOS}

Uma colônia abundante está sendo criada no insetário do laboratório a partir de 20 espécimes adultos coletados pela Fundação Nacional de Saúde sobre paredes rochosas sem associação com outras espécies de triatomíneos ou possíveis hospedeiros. O local de captura dos espécimes estava a cerca de $100 \mathrm{~m}$ de um domicílio no município de Itiquira, a $522 \mathrm{~m}$ de altitude e distante $347 \mathrm{~km}$ da capital (1708'02”S; 54²4'55”O).

Foi realizado um detalhado estudo morfológico das estruturas anatômicas cuticulares e da genitália externa do macho de 17 espécimes. Para os estudos da genitália, o $9^{\circ}$ segmento, ou pigóforo, foi retirado, aquecido em $\mathrm{KOH}$ a $10 \%$, e posteriormente dissecado em fenol, onde as estruturas analisadas permaneceram até a montagem final entre lâmina e lamínula em bálsamo do Canadá. Os desenhos foram confeccionados com auxílio de microscópio estereoscópico acoplado à câmara clara. Uma análise isoenzimática foi realizada sobre placa de acetato de celulose. Uma amostra de 17 espécimes da nova espécie foi comparada com 15 espécimes de T. jurbergi 21 exemplares de T. matogrossensis, as duas espécies do complexo morfologicamente mais próximas. Como base, 16 sistemas enzimáticos foram usados: aconitase (ACON, EC 4.2.1.3.), diaforase (DIA, EC 1.6.2.2.); frutose-1,6 difosfatase (FDP, EC 3.1.3.11.), fumarasa hidratase (FUM, EC 4.2.1.2.), glutamato desidrogenase (GDH, EC 1.4.1.3.), glutamato oxaloacetato transaminase (GOT, EC 2.6.1.1.); glucose-6- 
fosfato desidrogenase (G-6-PDH, EC 1.1.1.49.); glucose fosfato isomerase (GPI, EC 5.3.1.9.); alfa-glicerofosfato desidrogenase (GPD, EC 1.1.1.8.); hexokinase (HK, EC 2.7.1.1.), isocitrato desidrogenase (IDH, EC 1.1.1.42.); malato desidrogenase (MDH, EC 1.1.1.37.); enzima málica (ME, EC 1.1.1.40.); mannose-fosfato isomerase (MPI, EC 5.3.1.8.), fosfoglucomutase (PGM, EC 2.7.5.1.) e 6fosfogluconate desidrogenase (6-PGDH, EC 1.1.1.44.). As condições de migração e coloração são derivadas de Richardson et al. (1986) modificadas por Noireau et al. (1998).

\section{DESCRIÇÃO}

Triatoma vandae sp. $\mathrm{n}$.

Comprimento total médio (machos): $27.6 \pm 0.5 \mathrm{~mm}$ (mín. 27-máx. 28). Comprimento médio da cabeça: $5.5 \pm 0.5 \mathrm{~mm}$ (5-6). Comprimento médio do pronoto: $4.5 \pm 0.4 \mathrm{~mm}$ (4-5). Largura média da cabeça ao nível dos olhos: $2.5 \pm 0.1$ (22.5) $\mathrm{mm}$. Largura média do pronoto: $5.5 \pm 0.2 \mathrm{~mm}$ (5.5-6).

Cor geral castanho amarelado. Cabeça, pronoto, pleuras, prosterno e abdômen totalmente pretos. Hemélitros e parte dorsal das patas castanhos. Áreas ventrais das patas e do conexivo amarelas (Figs. 1a, 2a, b).

Cabeça preta, granulosa, pouco brilhante, 2.3 vezes mais longa que larga e mais longa que o pronoto (relação 1:0.73). Região anteocular 4.6 vezes mais longa que a pósocular (relação 1:0.22). Região pós-ocular com os lados quase retos e paralelos ou ligeiramente convergentes para trás. Clípeo da mesma largura que as genas em sua metade anterior e mais largo na parte posterior em vista dorsal. Genas ultrapassando o ápice do clípeo e moderadamente aguçadas. Jugas curvas apicalmente. Olhos atingindo mas

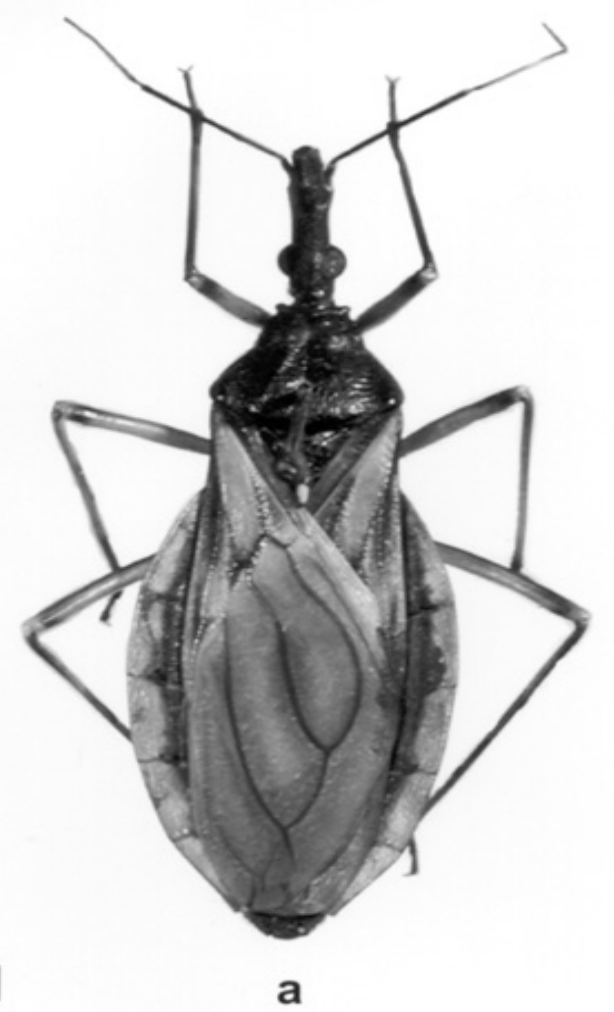

não ultrapassando o limite inferior da cabeça em vista lateral mas não atingindo o bordo superior. Dorsalmente, a proporção entre a largura de um olho e a região interocular (sinlipsis) é de 1:1.5. Tubérculos anteníferos localizados um pouco adiante da metade da região ante-ocular ao nível das jugas, atingindo o bordo inferior da cabeça que é mais delgada atrás do nível das jugas. Primeiro segmento antenal não atingindo o extremo anterior do clípeo e com pilosidade moderada. Segundo segmento com abundante pilosidade e cujo comprimento é maior que a metade da largura do segmento e fica apertada contra a superfície do mesmo. Terceiro segmento com micropilosidades douradas. Quarto segmento com micropilosidades e sensilas longas isoladas. A proporção entre os segmentos antenais é de 1:4.3:3.1:2.5. Primeiro segmento do rostro escuro; segundo e terceiro castanhos claros amarelados. Último segmento com densa e forte pilosidade preta de comprimento quase igual à largura do segmento. A relação dos segmentos do rostro é de 1:2.3:0.7.

Pronoto preto, pouco brilhante. Lobo anterior pouco rugoso e pouco granuloso, colar bem marcado com ângulos ântero-laterais longos, arredondados e divergentes. Tubérculos discais e laterais ausentes; nessa área de uma superfície lisa e opaca nascem as carenas submedianas que atingem quase o bordo posterior do pronoto. Lobo posterior tenuamente rugoso com ângulos humerais bem marcados, arredondados e ligeiramente levantados. Escutelo preto, pouco brilhante com corpo robusto e depressão rugosa; processo escutelar com dois terços do comprimento do corpo escutelar e rugas transversais bem marcadas. Sulco estridulatório com estrias finas.

Fig. 1a: Triatoma vandae sp.n.; b: Triatoma jurbergi Carcavallo, Galvão \& Lent, 1998 
Hemélitros apenas atingindo o bordo posterior do abdômen. Clavo e cório amarelos, com exceção das nervuras que são escuras. Membrana castanho cinza, enfumaçada.

Patas de cor uniforme, castanha amarelada, mais claras na região ventral com uma única mancha escura no ápice dos fêmures. Fêmures anteriores e posteriores sem tubérculos, fêmures medianos com tubérculos vestigiais. Tíbias I com fosseta esponjosa.

Abdômen preto e brilhante, convexo em vista ventral e com microestrias transversais. Pilosidade muito peque-

2

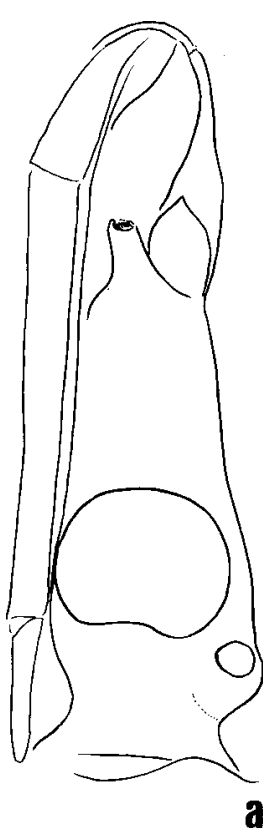

3

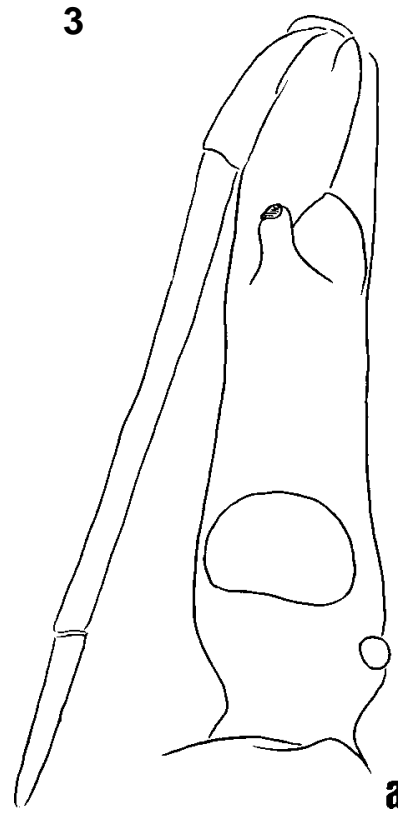

Fig. 2: Triatoma vandae sp.n. a: cabeça vista lateral; b: cabeça vista dorsal. Fig. 3: Triatoma jurbergi Carcavallo, Galvão \& Lent, 1998. a: cabeça vista lateral; b: cabeça vista dorsal. na e escassa. Espiráculos traqueais amarelos localizados quase sobre a sutura conexival. Conexivo ventral amarelo sem máculas, conexivo dorsal também amarelo, quase sem máculas com exceção de uma pequena linha preta intersegmental na união com a sutura.

A fêmea apresenta todos os caracteres descritos para o macho, entretanto, o comprimento total médio é maior: $29.5 \mathrm{~mm} \pm 0.5 \mathrm{~mm}$ (mín. 29-máx. 30).

Genitália do macho - Nono segmento constituído por uma cápsula arredondada/ovalada. Na sua porção terminal estão localizados superiormente o tubo anal constituído pelo $10^{\circ}$ segmento e o ânus propriamente dito que é o $11^{\circ}$ segmento. Abaixo destes está a abertura genital ladeada por $1+1$ placas, fracamente quitinizadas e móveis, que são os processos superiores do pigóforo. No bordo inferior existe uma projeção aguçada fortemente quitinizada que é o processo mediano do pigóforo. No processo superior do pigóforo estão localizados 1+1 parâmeros, órgãos auxiliares ao ato da cópula, que são cilíndricos, arqueados com numerosos pêlos longos na face externa e no bordo interno, com ápice curvado com projeção aguda (Figs. 7, 8, 10).

$\mathrm{O}$ aparelho articular é uma estrutura em forma da letra Y invertida, sendo que os braços divergentes constituem a base da estrutura; é denominado placa basal. Na região mediana externa está soldada a uma estrutura laminar retangular fendida longitudinalmente denominada extensão mediana da placa basal; abaixo dela, na face interna, se localiza uma estrutura cilíndrica, oca e aberta longitudinalmente, denominada processo do gonoporo e que se une nos dois extremos: (i) no superior ligado ao edeago e (ii) no inferior ligado a ponte basal, estrutura cilíndrica que se prende aos dois braços divergentes da placa basal (Figs. 4, 5, 6).

Edeago globoso, complexo constituído por estruturas quitinizadas e membranosas, a saber: uma placa externa laminar retangular de ápice arredondado que serve de assoalho ao conjunto denominado falosoma; uma estrutura cilíndrica fortemente quitinizada, interna, com base ímpar, de onde partem dois braços paralelos que se afastam e se tocam no ápice, denominada suporte do falosoma; uma membrana elástica denominada endosoma que ocupa todo o edeago e é invertida no ato da cópula. No endosoma estão diferenciadas duas estruturas: $1+1$ áreas laminares, situadas lateralmente, estriadas, aliformes com numerosos espinhos microscópicos situados no ápice: são os processos do endosoma; e uma estrutura localizada no ápice do endosoma, pouco quitinizada, com formato de um losango e denominada vesica (Figs. 9, 11, 12). Não foram observadas variações intraespecíficas nas estruturas fálicas (Jurberg et al. 1998).

Análises isoenzimáticas - Geneticamente, T. vandae é próxima de T. matogrossensis e sobretudo de T. jurbergi. $\mathrm{Na}$ análise isoenzimática possibilitando a interpretação de 18 loci gênicos, quatro loci diagnósticos ( $G d h$, Got, $M d h 1$ e $6 P g d h$ ) foram encontrados entre $T$. vandae e $T$. matogrossensis e dois loci permitiram diferenciar a nova espécie de T. jurbergi (Mdh2 e 6Pgdh). A proporção de loci polimórficos foi de $0.11 \mathrm{em}$ T. vandae (Acon e Pgm) e nula em T. jurbergi e T. matogrossensis. Na população analisada não foi mostrado nenhum desequilíbrio de 


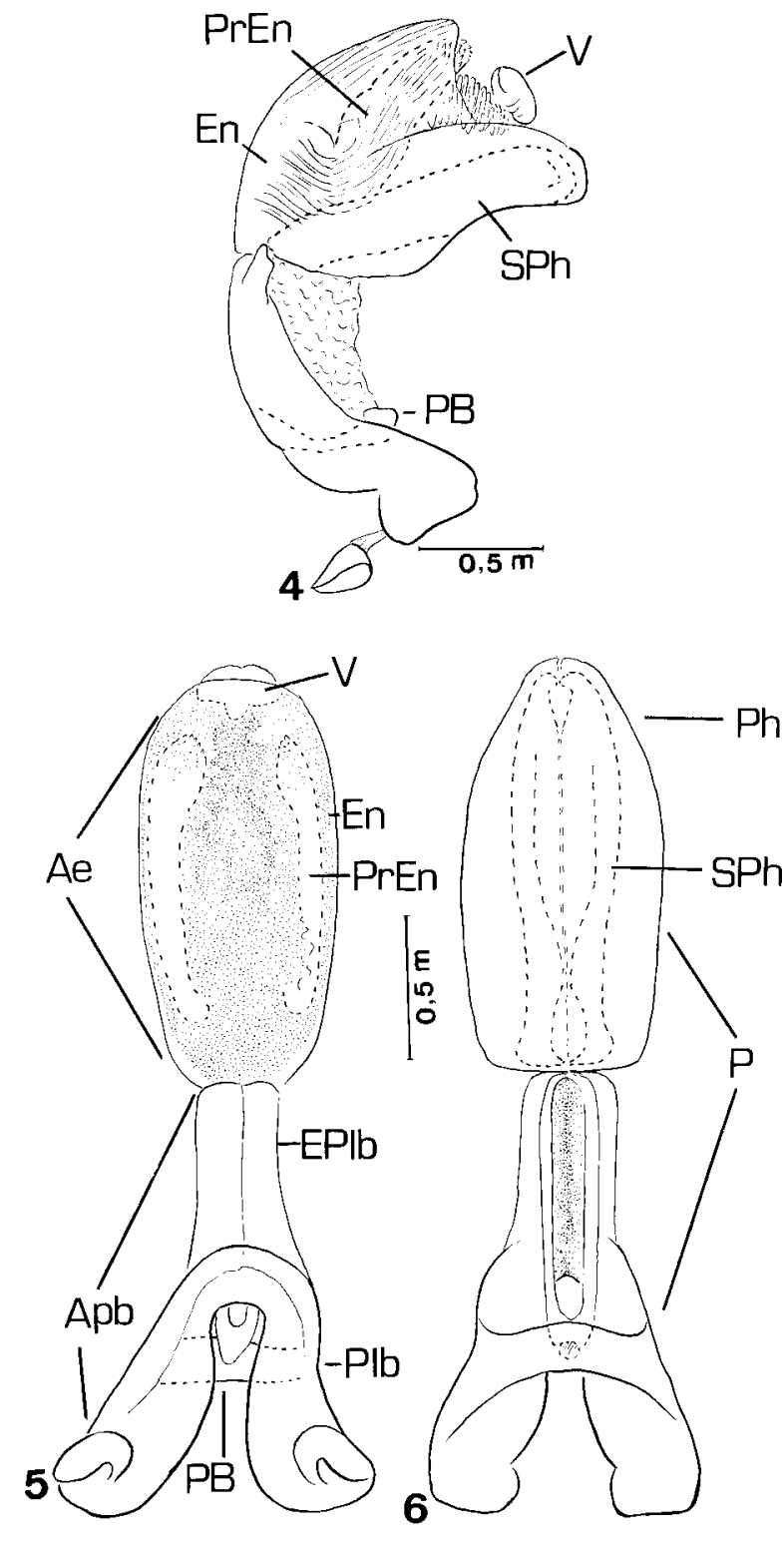

Triatoma vandae sp.n. Fig. 4: genitália do macho, falo em vista lateral. Fig. 5: genitália do macho, falo distendido em vista dorsal. Fig. 6: genitália macho, falo distendido em vista ventral (V: vesica; En: endosoma; PrEn: processo do endosoma; SPh: suporte do falosoma; Eplb: extensão mediana da placa basal; P: falo; Plb: placa basal; PB: ponte basal; Apb: aparelho articular; Ae: edeago; terminologia utilizada segundo Jurberg et al. 1998).

Hardy-Weinberg pelo cálculo dos indexes de fixação $\mathrm{F}$ (Nei 1987). A avaliação da diversidade gênica (He) foi de 0.03 para $T$. vandae. A distância genética de Nei entre $T$. vandae e $T$. jurbergi era 0.12 (0.25 entre $T$. vandae e $T$. matogrossensis).

Material tipo - Holótipo macho (no 5635), alótipo fêmea (n- 5636), 4 parátipos machos (n 으 5637, 5638, 5639, 5640 ) e 7 parátipos fêmeas (n오 $5641,5642,5643,5644$, 5645,5646 e 5647) procedentes de Itiquira, Mato Grosso, Brasil, estão depositados na coleção Rodolfo Carcavallo.
Dois parátipos machos (nos 2940,2941$)$ e dois parátipos fêmeas (n $\underline{\mathrm{OS}} 2942,2943$ ), da mesma procedência, estão depositados na coleção Herman Lent. Ambas coleções fazem parte do acervo da Coleção Entomológica do Instituto Oswaldo Cruz e estão sob a responsabilidade e guarda do Laboratório Nacional e Internacional de Referência em Taxonomia de Triatomíneos, Departamento de Entomologia, Instituto Oswaldo Cruz, Rio de Janeiro.

Diagnose - A espécie morfologicamente mais próxima é T. jurbergi Carcavallo, Galvão \& Lent, 1998, da qual se diferencia pela cor geral, pelas proporções das estruturas da cabeça, pela falta de tubérculos nos fêmures, e pelas patas uniformemente amareladas em todos os espécimes de T. vandae, é também uniformemente castanhoavermelhada na outra espécie. As estruturas da genitália externa masculina apresentam diferenças (Tabela). A nova espécie tem alguma semelhança cromática com $T$. williami Galvão, Souza \& Lima, 1965 e com T. baratai Carcavallo \& Jurberg, 2000. A coloração das ninfas é marrom em $T$. vandae e marrom-avermelhado em $T$. jurbergi, permitindo uma fácil diferenciação entre ambas as espécies (Fig. 1, 3,
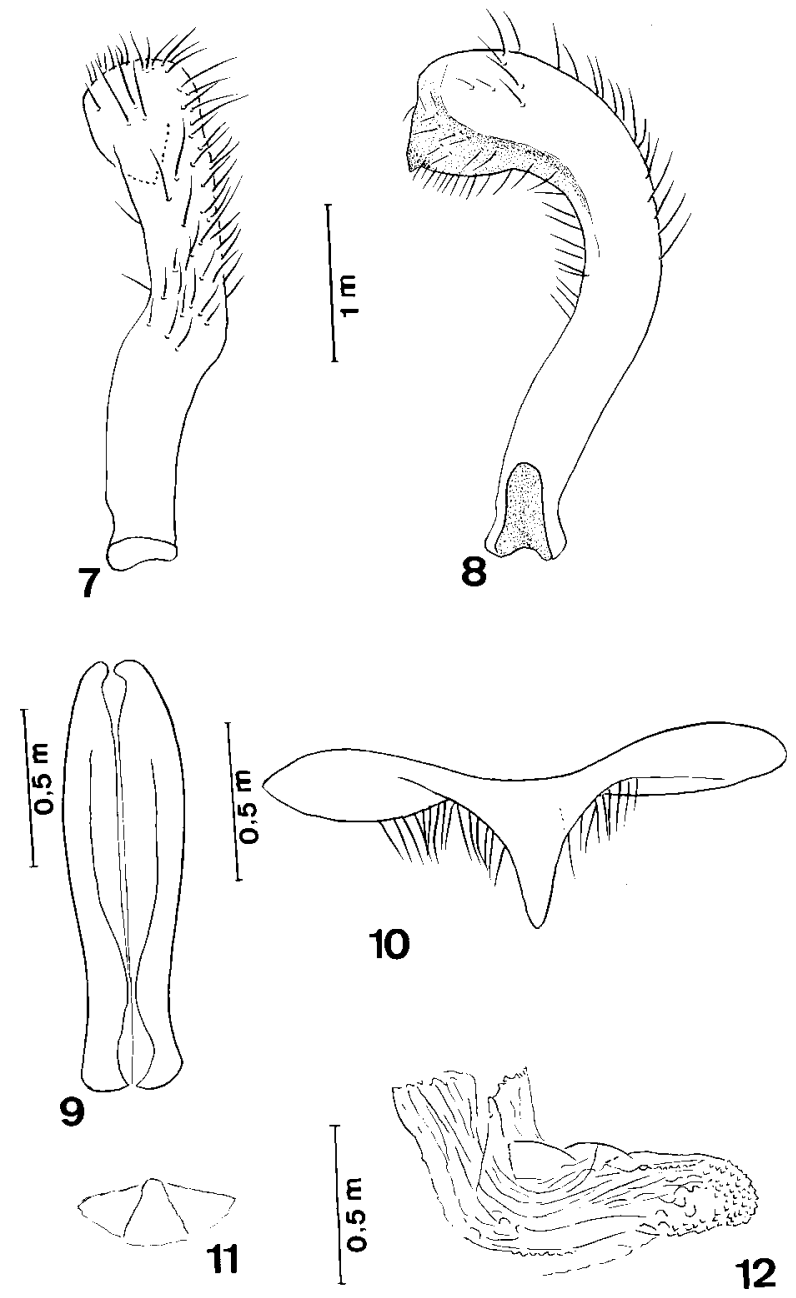

Triatoma vandae sp.n. Fig. 7: genitália do macho, parâmero, vista perfil. Fig. 8: genitália do macho, parâmero, vista ventral. Fig. 9: genitália do macho, suporte do falosoma. Fig. 10: genitália do macho, processo mediano do pigóforo. Fig. 11: genitália do macho, vesica. Fig. 12: genitália do macho, processo do endosoma. 
TABELA

Diferenças morfológicas entre Triatoma vandae sp. n. e Triatoma jurbergi Carcavallo, Galvão \& Lent, 1998

\begin{tabular}{lll}
\hline & T. vandae sp.n. & T. jurbergi Carcavallo, Galvão \& Lent, 1998 \\
\hline Cor geral dos adultos & Castanho amarelado; hemélitros castanhos; & Marron avermelhado; em quase todo o \\
patas (ventral) e conexivo com áreas amarelas & cório e com manchas conexivais alaranjadas \\
Cor geral das ninfas & Marrom & Marrom avermelhado \\
Comprimento cabeça/pronoto & $1: 0.73$ & $1: 0.58$ \\
Região ante-ocular/pós-ocular & 4.6 vezes ou mais & 6 vezes ou mais \\
Olho (dorsal)/sinlipsis & $1: 1.50$ & $1: 1.16$ \\
Segmentos rostrais & $1: 2.3: 0.7$ & $1: 3.2: 1$ \\
Pronoto & Preto & Castanho avermelhado \\
Tegumento do lobo anterior do pronoto & Pouco rugoso e granuloso & Bem marcado \\
Fêmures posteriores & Dentículos ausentes & Dentículos vestígiais \\
Conexivo dorsal & Quase sem máculas, com uma pequena linha & Com marcas intersegmentares bem \\
& preta na sutura (Fig. 1a) & evidentes (Fig. 1b) \\
Processo do endosoma & $\begin{array}{l}\text { Ápice arredondado coberto de dentículos } \\
\text { (Fig. 12) }\end{array}$ & $\begin{array}{l}\text { Ápice acuminado coberto de dentículos } \\
\text { (Fig. 11) (Carcavallo et al.1998) }\end{array}$ \\
Processo mediano do pigóforo & $\begin{array}{l}\text { Ápice acuminado, mediano base reta } \\
\text { (Fig. 10) }\end{array}$ & $\begin{array}{l}\text { Ápice acuminado curta, base arqueada. } \\
\text { Vesica }\end{array}$ \\
& $\begin{array}{l}\text { Fracamente quitinizada. Ápice acuminado } \\
\text { (Fig. 11) }\end{array}$ & $\begin{array}{l}\text { Ápice arredondado (Fig. 12) (Carcavallo } \\
\text { et al. 1998) }\end{array}$ \\
\hline
\end{tabular}

Tabela). Dois loci ( $M d h 2$ e $6 P g d h)$ foram encontrados como diagnósticos entre $T$. vandae e $T$. jurbergi, a espécie morfologicamente mais próxima. As duas populações analisadas eram procedentes de lugares distantes cerca de $110 \mathrm{~km}$. A distância genética de Nei, calculada a partir de 18 loci interpretados é baixa (0.12) mas não está em desacordo com a hipótese de duas espécies distintas (Dujardin et al. 2000, Dujardin comun. pessoal).

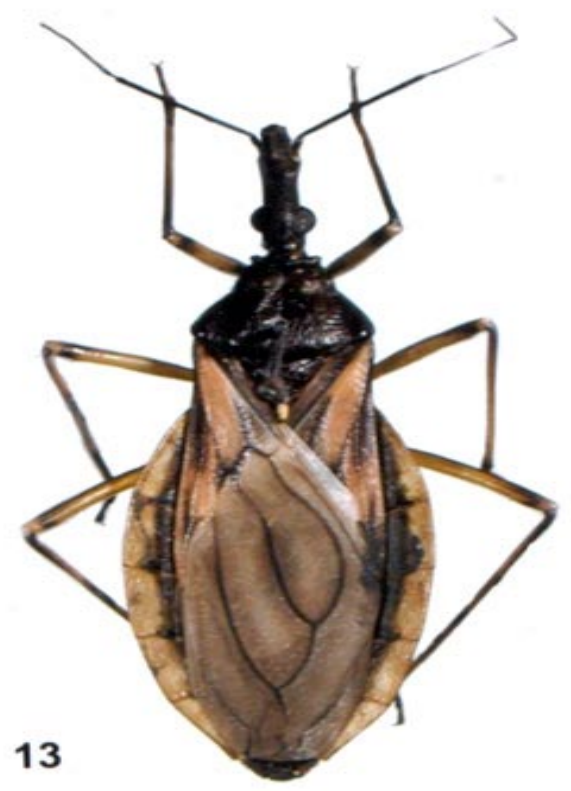

13: Triatoma vandae sp.n. vista da coloração geral.
Etimologia - O nome específico é uma homenagem a Sra. Vanda Cunha, técnica do laboratório que há aproximadamente 30 anos tem cuidado da colônia e auxiliado nos experimentos com triatomíneos.

\section{AGRADECIMENTOS}

Ao Sr. José Ferreira de Figueiredo, Fundação Nacional de Saúde de Mato Grosso, que coletou e enviou os exemplares da nova espécie para identificação dentro do Convênio Funasa/ Fiocruz. Ao Dr. Jean-Pierre Dujardin pelos comentários sobre a interpretação dos marcadores isoenzimáticos. A dois pareceristas anônimos pelos comentários e sugestões.

\section{REFERÊNCIAS}

Carcavallo RU, Jurberg J 2000. Triatoma baratai sp.n. do estado do Mato Grosso do Sul (Hemiptera, Reduviidae, Triatominae). Entomol Vect 7: 373-387.

Carcavallo RU, Galvão C, Lent H 1998. Triatoma jurbergi sp. n. do norte do Estado do Mato Grosso, Brasil (Hemiptera, Reduviidae, Triatominae) com uma atualização das sinonímias e outros táxons. Mem Inst Oswaldo Cruz 93: 459-464.

Carcavallo RU, Jurberg J, Lent H 1999. Phylogeny of the Triatominae. A - General approach. In RU Carcavallo, I Galíndez-Giron, J Jurberg, H Lent (eds.), Atlas of Chagas Disease Vectors in the Americas, Vol III, Fiocruz, Rio de Janeiro, p. 925-969.

Carcavallo RU, Jurberg J, Lent H, Galvão C, Steindel M, Pinto CJC 2001. Nova espécie do complexo oliveirai (nova denominação para o complexo matogrossensis) (Hemiptera, Reduviidae, Triatominae) do estado do Rio Grande do Sul, Brasil. Mem Inst Oswaldo Cruz 96: 71-79.

Carcavallo RU, Jurberg J, Lent H, Noireau F, Galvão C 2000. Phylogeny of the Triatominae (Hemiptera: Reduviidae). 
Proposals for taxonomic arrangements. Entomol Vec 7 (Supl. 1): 1-99.

Dujardin JP, Schofield CJ, Panzera F 2000. Les Vecteurs de la Maladie de Chagas. Recherches Taxonomiques, Biologiques et Génétiques, Academie Royale des Sciences d'Outre Mer, Brussels. Classe des Sciences Naturelles et Médicales NS 24 (5), 162 pp.

Galvão C, Rocha DS, Jurberg J, Carcavallo RU 2001. Ampliação da distribuição geográfica de Triatoma deaneorum Galvão, Souza \& Lima 1967, nova denominação para Triatoma deanei (Hemiptera, Reduviidae). Rev Soc Bras Med Trop 34: 587-589.

Jurberg J, Lent H, Galvão C 1998. Male genitalia and its importance in taxonomy. In RU Carcavallo, I Galíndez Girón, J Jurberg, H Lent (eds.), Atlas of Chagas Disease Vectors in the Americas, Vol. I, Fiocruz, Rio de Janeiro, p. 85-106.

Lent H, Wygodzinsky P 1979. Revision of the Triatominae (Hemiptera, Reduviidae), and their significance as vectors of Chagas disease. Bull Am Mus Nat Hist 163: 123-520.

Nei M 1987. Molecular Evolutionary Genetics, Columbia University Press, New York, 512 pp.

Noireau F, Gutierrez T, Zegarra M, Flores R, Brenière F, Cardozo L, Dujardin JP 1998. Cryptic speciation in Triatoma sordida (Hemiptera: Reduviidae) from the Bolivian Chaco. Trop Med Int Health 3: 364-372.

Richardson BJ, Baverstock PR, Adams M 1986. Allozyme Electrophoresis. A Handbookfor Animal Systematics and Population Studies, Academic Press, Orlando, $410 \mathrm{pp}$.

Usinger RL, Wygodzinsky P, Ryckman RE 1966. The biosystematics of Triatominae. Ann Rev Ent 11: 309-330. 